\title{
The importance of the definition of weight
}

\author{
Bar Varda \\ Science Education, Hebrew University, Jerusalem, Israel \\ barvaeda@zahav.net.il
}

\begin{abstract}
Presented by the Newton definition of weight that identifies the weight with the force of gravity exerted on the body, at the ninth grade, results in miss- conceptions: gravity stops at the end of the atmosphere and needs air for its activity, gravitation is unique to earth does not act in other locations such as on the moon or Mars. There is no gravity in space. An astronaut orbiting around the earth is not affected by gravity. When these findings were researched at high school: at grades eleven and twelve, among students and teachers the same miss- conceptions were observed. No reduction of them was found. Differentiation between apparent and real weight did not improve the results. Facing these results it was suggested to change the definition of weight and choose the one that young pupils use: weight is the force exerted by the body on the hand or any other support including the scale. This definition assumes that free falling bodies have no weight. In this way continuity is achieved both with young pupils' definition and the assumption made in general relativity that falling bodies have no weight. Two research works were made using this definition and resulted with a significant reduction of the miss-conception and even vanishing of some of them. This result was achieved using two different instruction methods.
\end{abstract}

Keywords: weight, mass, gravity, weight definition.

\section{Introduction}

Weight is a central concept in K-12 science education. However, it is unnecessarily confusing by a shift in the definition of weight typically introduced at the middle or high school level (Galili, 2001). This shift in the definition of weight is not arbitrary. It has roots in the history of science, both ancient and modern, which are important to understand in order to consider a modification in the curriculum. We therefore briefly summarize the history of the weight concept (Galili, 2001, 2012) as an introduction to this study. This shift was made since the definition of weight as the gravitation force exerted on the body results with miss- conceptions. Presented by the Newton definition of weight that identifies the weight with the force of gravity exerted on the body, at the ninth grade, results in the following miss-conception: gravity stops at the end of the actmosphere and needs air for its activity (Bar, Zinn and Rubin 1997), gravitation is unique to earth does not act in other locations such as on the moon or Mars (Brosh 2011, Ruggiero, Cartelli, Dupre, F, and Vincentini-Missoni, M. 1985). There is no gravity in space. An astronaut orbiting around the earth is not affected by gravity (the same). When these findings were researched at high school: at grades eleven and twelve, among students and teachers the same miss- conceptions were observed. No reduction of them was found (Galili and Kaplan 1996, Galili and Lehavi 2003). Differentiation between apparent and real weight did not improve the results.

Facing these results it was suggested to change the definition of weight and choose the one that young pupils use: weight is the force exerted by the body on the hand or any other support including the scale. This definition assumes that free falling bodies have no weight. In this way continuity is achieved both with young pupils' definition and the assumption made in general relativity that falling bodies have no weight. This definition also emphasizes the separation between the mass and the weight. 


\section{Historical background}

In previous papers (Bar and Zinn, 1998) mentioned the parallelism between the historical development of ideas and cognitive development has importance for the educational researcher, the science teacher, and the learner. Educational researchers can gain insight into the difficulties experienced by learners by considering how the acquisition of those concepts challenged generations of scientists. Knowledge of the historical develop of scientific ideas can help teachers recognize specific conceptual difficulties that their students encounter. Learners can also benefit from historical accounts of scientists of the distant past who may have shared some of their own ideas about the world, but which was changed as a result of new data, or new ways of thinking.

In the history of science, the weight concept developed prior to any understanding of mass or gravity. Two conceptions of weight are known from antiquity. Plato (428-327 BC) proposed that weight is a tendency of bodies to move towards similar bodies, so that a rock falls because it is attracted to other rocks. Aristotle (384-322 BC) included weight in his system of the world in which all heavy objects tended toward the center of the universe, which he took to be the center of the Earth. Both Aristotle and Plato included the idea of levity or lightness. In Aristotle's system, objects that were light, such as fire, tended to move upwards, away from the center of the universe. A generation later Euclid (325$265 \mathrm{BC}$ ) defined weight by the process of weighing something using a balance scale.

In renaissance times, Galileo (1564-1642 AD) discounted the idea of levity, countering Aristotle's idea that if you tied together a light object and a heavy object they would balance each other, by claiming that the sum of the two objects would be heavier than either one alone. The light body does not add lightness abs $d$ the summed weight is higher than either of the bodies Although Galileo supported Copernicus' idea that Earth revolved around the sun, Newton's idea of gravity had not yet been invented, so Galileo had no good explanation for how the Earth kept circling the sun, or why the moon didn't fall to Earth. So even as late as Galileo's time, the modern concepts of mass and gravity had not been invented yet.

The greatest change in the weight concept took place as part of the Newtonian revolution. In the Principia, Newton (1687) defined the force of attraction among all material objects as gravitation. He defined weight $(\mathrm{W})$ as the force of gravitation $(\mathrm{Fg})$ acting on a body and made a clear distinction between mass $(\mathrm{m})$ and weight that can be expressed in a simple equation relating these two quantities to the free acceleration $(\mathrm{g})$ towards the Earth:

$$
\mathrm{W}=\mathrm{F}_{\mathrm{g}}=\mathrm{mg}
$$

The concept of weight as defined by Newton was not a property of a single object, but rather a force between two objects.

It is important to note that throughout history - both before Newton and even today - people do not commonly use this definition. Instead, when asked what something weighs, they reply with what a spring scale would say if the object were weighed. They would not give the mass in grams or kilograms times 9.8 meters per second per second. It is only in science class, starting with the middle school level, and certainly in high school physics, where students are expected to give that answer.

Newton differentiated between weight and mass, but weight and gravity remained identified. In the general relativity became separated. This development supported a change in the definition of weight in the class. This change will be described here. 
The development of pupil's ideas

Young children associate the weight of an object with the effort to hold, lift, or move it. A study by Galili and Bar (1997) with 505 participants aged 10 to 16 found: Youngest children ages 5-6 (Kindergarten, $1^{\text {st }}$ grade) thought that an object was heaviest when it was in the form of a ball, and that it weighed less when it was deformed or cut into pieces. They expressed their understanding of weight in tactile terms, saying for example that heavy objects pressed hard on their hand, or were hard to hold or to lift. The ball-shaped object felt heavier when they held it in their hand, so they believed that it actually was heavier than when it was reshaped or divided. Very few of the children of this age attributed weight to light objects, such as a feather, a cotton ball, a hair, or dust.

Children aged 8-9 (second to third grade) about 80\% were able to conserve weight despite changes in appearance, recognizing that the weight of an object would not change if it is deformed or cut. In addition to expressing the idea of weight as how hard it is to hold or move an object - that is, a tactile response-many children of this age added the idea of weight as the "heaviness" of an object, which can be taken as a rough idea of mass. As shown by Bar et al. (1994), about age 8-9 most children gave "heaviness" of an object for the reason why it falls when released.

But, although the great majority of children at age 8-9 were able to conserve solid matter when it was deformed or cut, only about half recognized that the weight of a sample stayed the same when a solid became a liquid, as when ice melted or candle wax melted. The ability to conserve weight under change from solid to liquid did not occur for most subjects (about 75\%) until age 10 (fifth grade.) Children attributed weight to light objects, but still only $40 \%$ attributed any weight at all to a hair, and only $10 \%$ to dust. When asked whether or not a sample of matter weighed the same when it became a gas, as when water evaporated, a minority of 10 year-olds $(32 \%)$ were conservers. By age $13\left(8^{\text {th }}\right.$ grade) more than half were conservers $(59 \%)$, but it wasn't until age 15 or $16\left(11^{\text {th }}-12^{\text {th }}\right.$ grade) when the great majority of students $(80 \%)$ understood that the weight of a substance remained the same, whether a substance was deformed, changed to a liquid, or a gas. Also, it wasn't until age 15-16 when more than $90 \%$ of the subjects recognized that even very light objects, such as a hair or dust, have weight. However, even among 16 year-olds $17 \%$ of the students did not think that air has weight.

\section{Why do things fall?}

A study (Bar et al. 1994) involved individual interviews with 400 children age span 4-13. In that study the children were asked why things fall. Nearly all of their responses could be summarized in three categories: a) the object was not held; b) the object was heavy; and c) the object was pulled by the attractive force of the Earth (i.e. gravitation). As shown in Figure A, at the youngest ages the great majority of children responded that objects fall because they are not held up by something, hinting at the tactile definition of weight. Between ages 7 and 10 all three explanations were common. By the time they reached age 13, the great majority of children say that objects fall because they are pulled by the attractive force of the Earth (or by gravity). The changing frequency of responses as a function of age is shown in Figure A, This finding is consistent with instruction in the weight and mass concepts common in the U.S., Israel, and most developed nations. Most instructional materials introduce weight at the elementary level with an operational definition, as the process of weighing an object with a calibrated spring scale. At the middle school level the concept of mass is introduced, typically with the phrase "amount of matter." Mass is operationally defined and distinguished from weight by comparing the masses of two objects by use of a balance, it is explained that since this equilibrium does not change with location the mass is conserved. At this point students are given a new definition of weight as equivalent to the force of gravity on an object. Students are given the example that an object transported to the moon would weigh less since the Moon has only one sixth the amount of gravity as Earth, but the mass of the object would not change. 


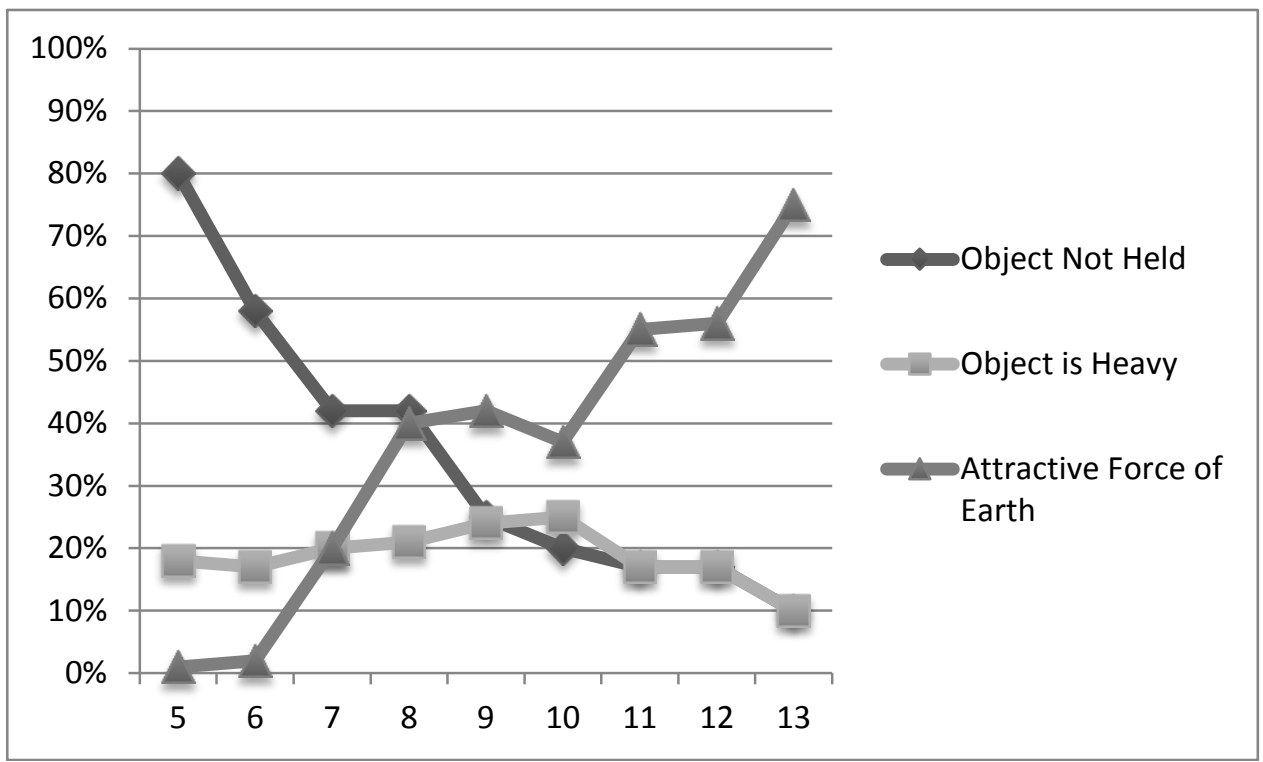

Figure 1. The reasons for free fall as given by children from age 5 to age 13. (Bar et al.1994).

This finding is consistent with instruction in the weight and mass concepts common in the U.S., Israel, and most developed nations. Most instructional materials introduce weight at the elementary level with an operational definition, as the process of weighing an object with a calibrated spring scale. At the middle school level the concept of mass is introduced, typically with the phrase "amount of matter." Mass is operationally defined and distinguished from weight by comparing the masses of two objects by use of a balance. At this point students are given a new definition of weight as equivalent to the force of gravity on an object. Students are given the example that an object transported to the moon would weigh less since the Moon has only one sixth the amount of gravity as Earth, but the mass of the object would not change. This findings of show that students' just entering high school as it suggests that students have some qualitative understanding of weight and gravity.

Palmer (2001) conducted individual interviews of 56 grade 6 (11-12 years-old) and 56 grade 10 (15-16 years old) in Australia. Students were asked to identify which objects were acted on by gravity in nine different scenarios and to justify their choices. Only 11\% of the students in grade 6 and 29\% of the students in grade 10 correctly indicated that gravity acted on all the objects. The students who indicated that gravity did not act in some of the situations gave a variety of answers. The most common of these were that gravity only acts on falling objects but not on objects moving upward ( $40 \%$ in grade 6 and $45 \%$ in grade 10 , of those who did not indicate that gravity acted in all nine scenarios); that gravity does not act on stationary objects "since they are not moving" ( $34 \%$ in grade 6 and $28 \%$ in grade 10); and that gravity does not act on objects buried underground ( $74 \%$ in grade 6 and $60 \%$ in grade 10). This finding is consistent with the finding by other researchers (Gunstone and Watts 1985) that force implies motion, and the direction of the force is parallel to the direction of the motion.

As students mature through the grades they are more likely to say that things fall because they are attracted by the Earth, or due to gravity. On the other hand few students seem to understand that gravity acts on all objects within a gravitational field, regardless of whether they are falling, thrown upwards, or as we'll also see later, stationary on a table. This finding suggests that students may indeed learn from their teachers to say that objects fall due to gravity, but their understanding of the term "weight" has changed little from the definition that they learned in elementary school. And if students fail to learn the meaning of weight, then what does this say about their understanding of the more advance term, mass? 
Observing this research we looked at the ideas of pupils just before they were presented to instruction

\section{Research}

Population and sample

The research was carried out at the beginning of the ninth grade, as a forward for an instruction experiment that checked the influence of the changed definition of the weight which will be referred to as the operative definition since it uses the operation of weighing. The sample was 186 Participants, aged 14. The aim of the research was to find out the pre-conceptions of 14 years old participants before being surfaced to instruction; the research was carried out according the constructive theory of science education. Pre conceptions of the participants should be taken into account while constructing the experiences presented during the instructions.

Test

Q1. What is the meaning of weight?

a. If the object is big or small

b. If the object is heavy or light

c. The force of gravity exerted on the body

d. The quantity of matter the body contains

e. The force that the body exerts on its support

Q2. If a $5 \mathrm{~kg}$ object is taken to the Moon:

a. Both its weight and mass will increase.

b. Its mass will not change, but its weight will increase.

c. Its mass will not change, but its weight will decrease.

d. Both its mass and weight will decrease

Q3. An astronaut is in a space shuttle revolving around the Earth. In this situation:

a. The astronaut DOES have weight and IS influenced by force of gravity.

b. The astronaut does NOT have weight, and IS influenced by gravity.

c. The astronaut DOES have weight, and is NOT influenced by gravity.

d. The astronaut does NOT have weight, and is NOT influenced by force of gravity.

Q4. An object is falling to the moon's surface. In this situation:

a. The object DOES have weight and IS influenced by force of gravity.

b. The object does NOT have weight, and IS influenced by gravity.

c. The object DOES have weight, and is NOT influenced by gravity.

d. The object does NOT have weight, and is NOT influenced by force of gravity.

Q5. An object is in a free falling elevator. In this situation:

a. The object DOES have weight and IS influenced by force of gravity.

b. The object does NOT have weight, and IS influenced by gravity.

c. The object DOES have weight, and is NOT influenced by gravity.

d. The object does NOT have weight, and is NOT influenced by force of gravity.

Q6. An object is falling from a table. In this situation:

a. The object DOES have weight and IS influenced by force of gravity.

b. The object does NOT have weight, and IS influenced by gravity.

c. The object DOES have weight, and is NOT influenced by gravity.

$\mathrm{d}$. The object does NOT have weight, and is NOT influenced by force of gravity.

Q7. An object is resting on a table. In this situation:

a. The object DOES have weight and IS influenced by force of gravity.

b. The object does NOT have weight, and IS influenced by gravity.

c. The object DOES have weight, and is NOT influenced by gravity.

d. The object does NOT have weight, and is NOT influenced by force of gravity.

Q8. An object is resting on the moon's surface. In this situation:

a. The object DOES have weight and IS influenced by force of gravity.

b. The object does NOT have weight, and IS influenced by gravity.

c. The object DOES have weight, and is NOT influenced by gravity.

$\mathrm{d}$. The object does NOT have weight, and is NOT influenced by force of gravity. 


\section{Findings}

Q1. What is the meaning of weight? $49 \%$ of the participants choose: b. "If the object is heavy or light". During the class discussion that followed administration of the questionnaire students gave examples of heavy objects that are hard to hold and lift in order to illustrate the meaning that they attached to the word "weight." Thus, their ideas of weight included both the idea of "heaviness" and tactile forces-the feeling of a heavy or light object. They also said that a heavy body, the one that has high weight is also hard to move. This feature relates to weight through friction. We note that the most common response: $b$. "If the object is heavy or light" is ambiguous. That is, "heaviness" could be considered a naïve notion of weight as a property of a body, similar to the more formal concept of mass, or as a force that is exerted on a support, such as the felt weight of an object when held in the hand. This answer is consistent with the response of a substantial minority of students who explain that things fall "because they are heavy" (Bar et al. 1994), as well as other findings, such as the idea that astronauts must have heavy shoes to keep them on the moon where there is no gravity (Watts 1982). Without interviewing students it is difficult to tell which of these schemas are guiding their responses.

Q2. If a $5 \mathrm{~kg}$ object is taken to the Moon. The question is aimed at finding out whether or not the participants recognize that weight can change but mass does not change in a world with weaker gravity. Most of 14 years old pupils agreed that the weight will reduce while the mass will stay the same, consistent with DePierro and Garafalo (2003), who used the students' knowledge of this distinction in Socratic dialog as a means for advancing their understanding. This finding can be interpreted in view of the results of Q4: $45 \%$ said that the moon has gravity, for those who identify weight and gravity $(27 \%)$ it is natural to assume that the weight on the moon is diminished a or reduced.

Q3. An astronaut is in a space shuttle revolving around the Earth. The pupils are asked whether or not the astronaut has weight and is influenced by gravity. Only a minority of the students believe there IS gravity students believe that is the case (37\%). Regarding weight, the findings are reversed. About $50 \%$ believe that the astronaut has weight,.

Regarding gravity according to questions Q3 Q4 Q5 and Q6 the percentages incresed from 12 percents gradually to 50,63, and 90 percents. Most of the pupils agree that there is gravitation force in at the earth's surface; in the case of the falling elevator many students decided that there is no force acting in this situation. As mentioned 50 percent attributed gravitation force to the moons' surface, but only 12 percents said that the force of gravity acts on the revolving satellite. These ideas confirm with the schema that there is no gravity in space. The earth may be unique in its gravitation force. In contrast, we do not find the same relationship between location and weight for falling objects, where students that falling objects have weight on Earth's surface (65\%), in a falling elevator $(67 \%)$, on the moon $(61 \%)$, and in orbit (53\%)this finding show that about half of the participants think that falling bodies have weight. We do find, however, that pupils are more likely to say that an object at rest has weight. Contrast the numbers above with at rest on Earth $(86 \%)$ and even at rest on the moon $(71 \%)$. The case of the moon is especially interesting. The great majority of students who believe the moon has gravity also believe that an object resting on the moon has weight; but $26 \%$ of the students who believe there is no gravity on the moon also believe that an object resting on the moon's surface has weight.

To sum up these findings: During the critical period of upper elementary to middle school, very few students learn that gravity acts throughout space. Misconceptions about gravity are widespread, and most students enter intermediate school with the pre-Newtonian idea that gravity is a special property of planet Earth. Whether or not gravity is present, it is also influenced by the motion of the 
body depends on how that body is moving. The effort to define the property of weight as the force of gravity on an object is understood by almost no students. Instead, the idea of weight as "heaviness" of a body, which is more an operational definition of mass, remains for most students the central idea of weight; so that when they encounter the formal definition of mass at the intermediate school level, it is likely that they will have a difficult time separating the weight and the mass concepts. Failure to understand these three threshold concepts - weight, mass, and gravity -is likely to impede students' abilities to achieve performance expectations at high school, which assume students have a scientific conception of these concepts. The instruction will answer the problem how conventional instruction affects the miss- conception.

\section{References}

Bar, V., Zinn, B. and Rubin, E. (1997), Similar frameworks of action-at-a distance: Early scientist's and pupils' ideas, Science $\mathcal{E}$ Education, 7(5), 471-491.

Bar, V., Zinn, B., (1998). Similar frameworks of action-at-a-distance: Early scientists' and pupil's ideas. Science Education 7, 471491.

Bar, V., Zinn, B., Goldmuntz, R., and Sneider, C. (1994). Children's concepts about weight and free fall. Science Education, 78(2), 149-169.

Depiero and Garafalo (2003). Using a Socratic Dialog To Help Students Construct Fundamental Concepts. Journal of chemical education, 80(12), 1408.

Galili, I. and Kaplan, D. (1996). Students' Operation with the Concept of Weight. Science Education, 80(4), 457-487.

Galili, I. and Lehavi, Y. (2003). The importance of weightlessness and tides in teaching gravitation. American Journal of Physics, 71(11), 1127-1135.

Galili, I. (1993). Weight and Gravity: teachers' ambiguity and students' confusion about the concepts. International Journal of Science Education, 15(1), 149-162.

Galili, I. (2001). Weight versus gravitational force: Historical and educational perspectives. International Journal of Science Education, 23, 1073-1093.

Galili, I. (2011). 'Promotion of Content Cultural Knowledge through the use of the History and Philosophy of Science', Science $\mathcal{E}$ Education, DOI 10.1007/s11191-011-9376-x

Watts (1982). Gravity don't take it for granted. Physics Education, 17(4), 116. 